\title{
Chronically Activated T-cells Retain Their Inflammatory Properties in Common Variable Immunodeficiency
}

\author{
Roos-Marijn Berbers ${ }^{1} \cdot$ M. Marlot van der Wal ${ }^{2}$. Joris M. van Montfrans ${ }^{3} \cdot$ Pauline M. Ellerbroek $^{4}$. \\ Virgil A. S. H. Dalm ${ }^{5,6} \cdot$ P. Martin van Hagen ${ }^{5,6} \cdot$ Helen L. Leavis $^{1} \cdot$ Femke van Wijk $^{2}$
}

Received: 30 April 2021 / Accepted: 7 June 2021 / Published online: 11 July 2021

(c) The Author(s) 2021

\begin{abstract}
Purpose Immune dysregulation complications cause significant morbidity and mortality in common variable immunodeficiency (CVID), but the underlying pathophysiology is poorly understood. While CVID is primarily considered a B-cell defect, resulting in the characteristic hypogammaglobulinemia, T-cells may also contribute to immune dysregulation complications. Here, we aim to further characterize T-cell activation and regulation in CVID with immune dysregulation (CVIDid).

Methods Flow cytometry was performed to investigate T-cell differentiation, activation and intracellular cytokine production, negative regulators of immune activation, regulatory T-cells (Treg), and homing markers in 12 healthy controls, 12 CVID patients with infections only (CVIDio), and 20 CVIDid patients.

Results Both CD4 + and CD8 + T-cells in CVIDid showed an increased activation profile (HLA-DR +, Ki67 +, IFN $\gamma+$ ) when compared to CVIDio, with concomitant upregulation of negative regulators of immune activation PD1, LAG3, CTLA4, and TIGIT. PD1 + and LAG3 + subpopulations contained equal or increased frequencies of cells with the capacity to produce IFN $\gamma$, Ki67, and/or GzmB. The expression of PD1 correlated with serum levels of CXCL9, 10, and 11. Treg frequencies were normal to high in CVIDid, but CVIDid Tregs had reduced CTLA-4 expression, especially on CD27 + effector Tregs. Increased migratory capacity to inflamed and mucosal tissue was also observed in CVIDid T-cells.

Conclusion CVIDid was characterized by chronic activation of peripheral T-cells with preserved inflammatory potential rather than functional exhaustion, and increased tissue migratory capacity. While Treg numbers were normal in CVIDid Tregs, low levels of CTLA-4 indicate possible Treg dysfunction. Combined studies of T-cell dysfunction and circulating inflammatory proteins may direct future treatment strategies.
\end{abstract}

Keywords Immune dysregulation · Common variable immunodeficiency (CVID) · T-cells · Immune exhaustion · Regulatory T-cells $\cdot$ Autoimmunity

Helen L. Leavis, Femke van Wijk contributed equally to this work

Helen L. Leavis

h.leavis@umcutrecht.nl

1 Department of Rheumatology and Clinical Immunology, University Medical Center and Utrecht University, Utrecht, The Netherlands

2 Center for Translational Immunology, University Medical Center Utrecht and Utrecht University, Utrecht, The Netherlands

3 Department of Pediatric Immunology and Infectious Diseases, University Medical Center Utrecht and Utrecht University, Utrecht, The Netherlands
4 Department of Internal Medicine and Infectious Diseases, University Medical Center Utrecht and Utrecht University, Utrecht, The Netherlands

5 Department of Internal Medicine, Division of Clinical Immunology, Erasmus MC University Medical Center, Rotterdam, The Netherlands

6 Department of Immunology, Academic Center for Rare Immunological Diseases (RIDC), Erasmus MC University Medical Center, Rotterdam, The Netherlands 


\section{Introduction}

Common variable immunodeficiency (CVID) is characterized by recurrent infections caused by low IgG, and $\operatorname{IgA}$ or IgM [1], for which patients are treated with immunoglobulin G replacement therapy (IgRT) [2]. IgRT has significantly decreased the risk of infectious complications in CVID, but nonetheless, over a third of patients develop immune dysregulation complications [3,4], resulting in significant morbidity and mortality [5, 6]. A wide range of immune dysregulation phenomena can be observed in CVID, including granulomatous-lymphocytic interstitial lung disease (GLILD), enteritis, autoimmune cytopenias, lymphoproliferation, and hematological malignancies $[4,7]$. The underlying pathophysiology of immune dysregulation in CVID is currently poorly understood, which complicates diagnostics and treatment $[8,9]$.

While the defining hypogammaglobinemia in CVID is considered to be primarily the result of B-cell dysfunction, several lines of evidence suggest an additional role for T-cells in CVID with immune dysregulation (CVIDid). Biopsies of lung granulomas in CVIDid show predominance of CD4 + T helper $(\mathrm{Th})$ cells $[10,11]$, while regulatory T-cells (Tregs) are often absent [10]. In peripheral blood of patients with CVIDid, a decreased CD4/CD8 ratio was observed with decrease of naïve T-cells [12], Tregs, Th17 cells, and follicular helper T (Tfh) cells [13, 14]. Moreover, there are indications that CVID T-cells may be functionally exhausted, including reduced capacity to respond to bacterial antigens and increased expression of PD1 $[15,16]$. Our group and others have previously demonstrated that serum cytokines in CVIDid $[17,18]$ are shifted towards a Th1 phenotype, and we observed an upregulation of proteins associated with immune regulation-IL10, LAG3, and 4-1BB. Monogenic primary immunodeficiencies caused by mutations in immune regulation genes such as CTLA4 [19] and ICOS [20] often result in a CVIDid phenotype. However, how the interplay between immune regulation and immune activation results in CVIDid remains poorly understood.

To further study the balance between immune activation and immune regulation in CVIDid, we used flow cytometry to evaluate naïve T-cell subsets, T-cell activation and cytokine production, exhaustion, negative regulators of immune activation, regulatory T-cells, and T-cell homing markers.

\section{Methods}

\section{Ethics Statement}

Ethical approval for this study for all participants was received from the Medical Ethical Committee of the
Erasmus MC University Medical Center in Rotterdam, The Netherlands (METC: 2013-026). Written informed consent was obtained from all patients and controls according to the Declaration of Helsinki.

\section{Study Population and Sample Collection}

Patients were diagnosed with CVID according to the European Society for Immunodeficiencies criteria [1] and were included at the outpatient clinics of the UMC Utrecht and the Erasmus MC University Medical Center Rotterdam, The Netherlands. Patients were eligible if they were aged seven or older, and they and/or their legal guardians signed informed consent. Household members of patients were recruited as healthy controls (HC). Medication use up to 3 months prior to sampling was recorded.

\section{Sample Processing}

Peripheral blood mononuclear cells (PBMC) were isolated from blood by Ficoll density centrifugation (GE HealthcareBiosciences, $\mathrm{AB}$ ), and frozen at $-180{ }^{\circ} \mathrm{C}$ until use. Cells were subsequently thawed, counted and plated at 1,000,000 live cells per panel per sample. For the panels including intracellular cytokine measurement, cells were first restimulated with $20 \mathrm{ng} / \mathrm{mL}$ phorbol 12-myristate 13-acetate (PMA, MilliporeSigma) and $1 \mu \mathrm{g} / \mathrm{mL}$ ionomycin (MilliporeSigma) for $4 \mathrm{~h}$ at $37^{\circ} \mathrm{C}$ with addition of monensin (Golgistop, BD Biosciences, 1:1500) during the last $3.5 \mathrm{~h}$. Cell death was stained in all panels using Fixable Viability Dye eFluor 506 (eBioscience). Next, cells were incubated with the surface antibodies (Supplementary Table 1) for $20 \mathrm{~min}$ at $4{ }^{\circ} \mathrm{C}$ and washed. Cells were then permeabilized with fixation/permeabilization reagent (eBioscience) for $30 \mathrm{~min}$ at $4{ }^{\circ} \mathrm{C}$, washed, and incubated with the intracellular antibodies (Supplementary Table 1). Cells were stored at $4{ }^{\circ} \mathrm{C}$ until the next day, when they were measured on the LSR Fortessa (BD Biosciences).

\section{Analysis and Statistics}

For flow cytometric data, median fluorescence intensities (MFI) and percentages of positive cells were analyzed in FlowJo. All statistical analyses and graphic representations were done in R 3.2.0 [21]. Continuous variables were compared using the Mann-Whitney rank test, or paired Wilcoxon-rank test for paired samples. Correlation was calculated using Spearman's correlation. $\mathrm{P}$ values below 0.05 were considered statistically significant. 


\section{Results}

\section{The Overall T-cell Profile of CVIDid Patients Differs from CVIDio and HC}

To investigate immune activation and regulation in CVID, PBMC were isolated from 12 healthy controls (HC), 12 CVID patients with infections only (CVIDio), and 20 patients with CVIDid (Table 1). Patients were selected from a cross-sectional Dutch primary immunodeficiency cohort when they received IgRT and did not use any immunomodulatory medication during and the last 3 months prior to sampling. Three of the CVIDid patients had a known CVIDassociated monogenetic disease (CTLA4 haploinsufficiency, STAT1 gain of function, and PIK3R1). Flow cytometry was performed as described in the Supplementary Information (see also Supplementary Table 1 and Supplementary Figs. 1-4).

First, pooled flow cytometry data was analyzed in an unsupervised manner using principal component analysis (PCA, Fig. 1A). CVIDio and HC clustered closely together and were distinct from most CVIDid samples. This suggests that most variation in the flow cytometric T-cell data related to immune dysregulation and not to the hypogammaglobulinemia shared by CVID patients. CVIDid patients did not cluster by treatment history or location of autoimmunity (Supplementary Fig. 5), suggesting that peripheral blood T-cell skewing may be generalized among CVIDid patients.

\section{CVIDid T-Cells Are Th-1-Skewed and Chronically Activated}

Next, the distribution of CD4 + and CD8 + T-cell subsets in CVIDid was assessed. Frequencies in $\mathrm{CD} 4+, \mathrm{CD} 8+$, and naïve/memory subsets were similar to that reported in previous studies [12, 22]: a decreased CD4/CD8 ratio (Fig. 1B), and a trend of decreased naïve $(\mathrm{CD} 45 \mathrm{RA}+\mathrm{CCR} 7+)$ CD4+ T-cells in CVIDid compared to CVIDio $(p=0.07)$ (Fig. 1C). In addition, a subset of CVIDid patients showed a high percentage of $\mathrm{CD} 4+\mathrm{T}$ effector memory cells, and $\mathrm{CD} 4+\mathrm{T}$ effector memory cells re-expressing CD45RA (TEMRA), which are associated with chronic activation such as observed in viral infections [23] (Fig. 1C). Within the naïve CD4 + T-cells, CD31 + recent thymic emigrants

Table 1 Cohort characteristics. HC, healthy control; CVIDio, CVID with infections only; CVIDid, CVID with immune dysregulation; IQR, interquartile range; $D M A R D$, disease modifying anti-rheumatic drug; $V U S$, variant of unknown significance

\begin{tabular}{|c|c|c|c|}
\hline Summary statistics & $\mathrm{HC}(\mathrm{n}=12)$ & CVIDio $(n=12)$ & CVIDid $(n=20)$ \\
\hline \multicolumn{4}{|l|}{ Characteristics } \\
\hline Age (median, IQR) & $45.50(40.25,52.00)$ & $38.50(29.00,58.25)$ & $38.50(35.75,43.50)$ \\
\hline Sex (male) & $5(41.67 \%)$ & $4(33.33 \%)$ & $11(55.00 \%)$ \\
\hline Center (Utrecht) & $9(75.00 \%)$ & $11(91.67 \%)$ & $15(75.00 \%)$ \\
\hline Antibiotics & $0(0.00 \%)$ & $3(25.00 \%)$ & $8(40.00 \%)$ \\
\hline Immunosuppressive medication & $0(0.00 \%)$ & $0(0.00 \%)$ & $0(0.00 \%)$ \\
\hline $\operatorname{IgA}<0.1 \mathrm{~g} / \mathrm{L}$ & $0(0.00 \%)$ & $2(16.67 \%)$ & $13(65.00 \%)$ \\
\hline \multicolumn{4}{|l|}{ Immune dysregulation complications } \\
\hline Pulmonary & $0(0.00 \%)$ & $0(0.00 \%)$ & $8(40.00 \%)$ \\
\hline Hematological & $0(0.00 \%)$ & $0(0.00 \%)$ & $2(10.00 \%)$ \\
\hline Gastrointestinal & $0(0.00 \%)$ & $0(0.00 \%)$ & $8(40.00 \%)$ \\
\hline Rheumatological & $0(0.00 \%)$ & $0(0.00 \%)$ & $6(30.00 \%)$ \\
\hline Dermatological & $0(0.00 \%)$ & $0(0.00 \%)$ & $4(20.00 \%)$ \\
\hline Hematological malignancy & $0(0.00 \%)$ & $0(0.00 \%)$ & $2(10.00 \%)$ \\
\hline Lymphoproliferation (incl splenomegaly) & $0(0.00 \%)$ & $0(0.00 \%)$ & $10(50.00 \%)$ \\
\hline Other & $0(0.00 \%)$ & $0(0.00 \%)$ & $4(20.00 \%)$ \\
\hline DMARD-naïve/subclinical disease & NA & NA & $12(60.00 \%)$ \\
\hline Disease in remission & NA & NA & $8(40.00 \%)$ \\
\hline \multicolumn{4}{|l|}{ Genetics } \\
\hline Not done & $12(100.00 \%)$ & $12(100.00 \%)$ & $12(60.00 \%)$ \\
\hline Nothing found & $0(0.00 \%)$ & $0(0.00 \%)$ & $2(10.00 \%)$ \\
\hline VUS found & $0(0.00 \%)$ & $0(0.00 \%)$ & $2(10.00 \%)$ \\
\hline Pathogenic mutations found & $0(0.00 \%)$ & $0(0.00 \%)$ & $\begin{array}{l}3(15.00 \%)+1 \\
\text { TNFRSF13B (TACI) } \\
\text { mutation }\end{array}$ \\
\hline
\end{tabular}



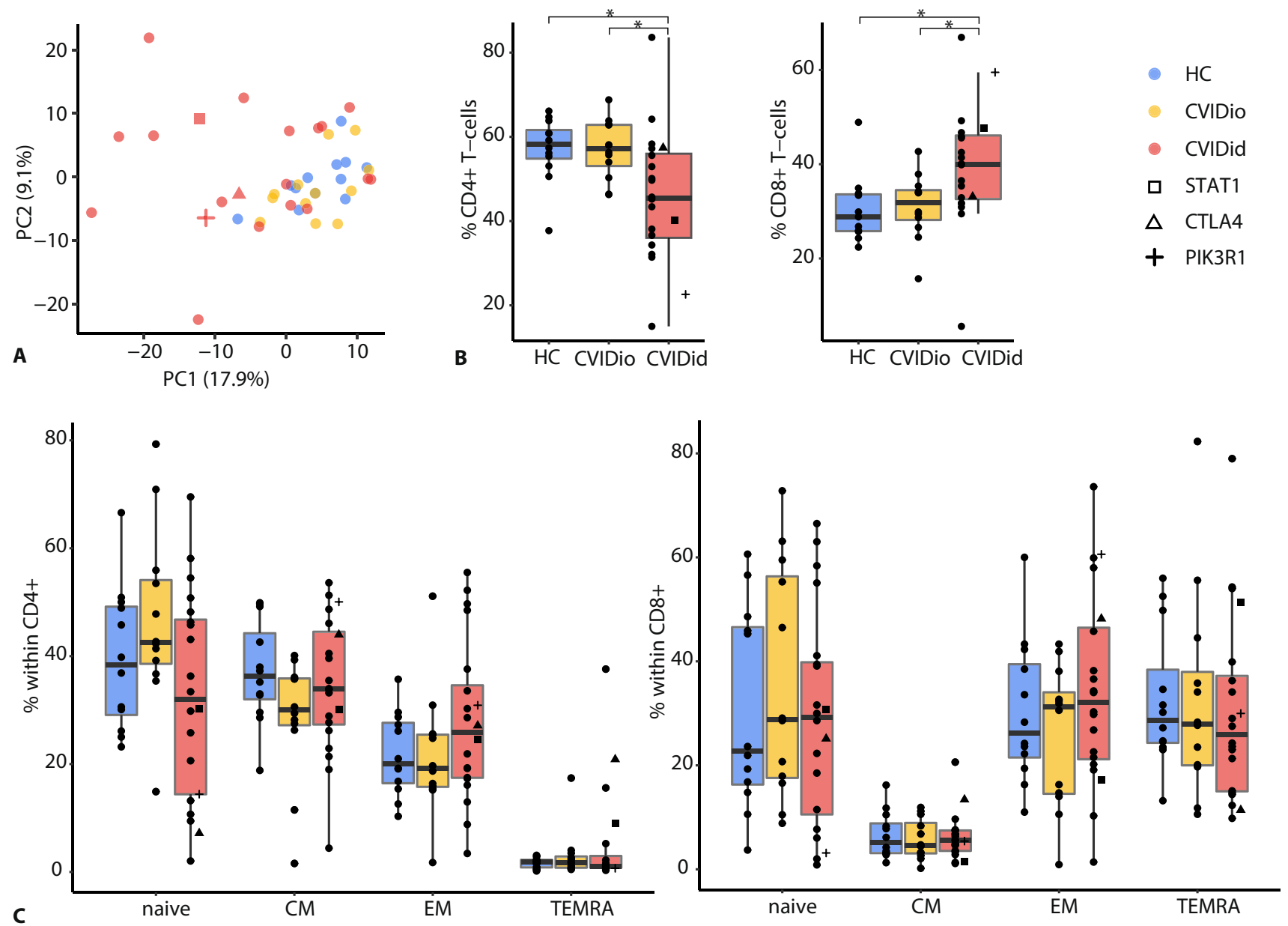

Fig. 1 General description of T-cell subsets in CVID. A Principal component analysis of FACS data (all panels combined). B CD4+and CD8+T-cells. C Naïve (CD45RA+CCR7+), central memory (CM: CD45RA-CCR7+), effector memory (EM: CD45RA-CCR7-) and terminally differenti-

ated effector memory cells (TEMRA: CD45RA+CCR7-) in CD4 + T-cells. CVIDid =CVID with immune dysregulation $(n=20)$, CVIDio $=$ CVID with infections only $(n=12), \mathrm{HC}=$ healthy controls $(\mathrm{n}=12)$. Statistics: Mann-Whitney U-test. $* \mathrm{p}<0.05, * * \mathrm{p}<0.01$, $* * * \mathrm{p}<0.001$

were more abundant in CVIDid than the CD31-central naïve T-cells (Supplementary Fig. 6). No differences in CD8 + T-cell distribution were observed (Fig. 1C).

Within the effector/memory $(\mathrm{CD} 45 \mathrm{RO}+)$ subpopulation, proportions of CD4 + T-cells expressing HLA-DR, Ki67, and IFN $\gamma$ were significantly increased in CVIDid, while IL17a, IL13, and TNF- $\alpha$ expressing T-cells were not different between CVIDid and CVIDio (Fig. 2A). A similar activation pattern was observed in the CD8 + effector memory population for HLA-DR, Ki67, and GzmB (Fig. 2B). We previously described [17] serum cytokine and chemokine levels in an overlapping cohort, allowing comparison between soluble serum markers and T-cell characteristics. Pooling the data from HC, CVIDio, and CVIDid, we observed that the proportion of IFN $\gamma+$ Th cells correlated with serum levels of interferon-inducible chemokines CXCL9, 10, and 11 (Fig. 2C). While we observed an increase of serum IL17a in
CVIDid, there was no corresponding increase of IL17a-producing Th cells, and the frequency of these cells did not correlate with the serum IL17a levels (Supplementary Fig. 7).

\section{T-cells Expressing Negative Regulators of Immune Activation Retain Their Inflammatory Potential in CVIDid}

Next, we investigated whether this immune activation resulted in immune exhaustion in CVIDid, which is known to happen in the context of chronic inflammation [24]. In CVIDid CD4 +-cells, we observed an increased proportion of cells expressing negative regulators of immune activation PD1, LAG3, CTLA4, ICOS, and TIGIT (Fig. 3A). In addition, CVIDid CD4 + T-cells expressed higher levels of CD95 (FAS-L), showing that they are terminally differentiated and may be more prone to apoptosis (Fig. 3B). In 
Fig. 2 Expression of activation markers HLA-DR, Ki67, and $\mathrm{GzmB}$, and of intracellular cytokines after PMA/ ionomycin stimulation: IFNg, TNFa, IL-13, and IL-17a. A $\mathrm{CD} 4+\mathrm{CD} 45 \mathrm{RO}+\mathrm{T}$-cells. B CD8 + CD45RO + T-cells. CVIDid $=$ CVID with immune dysregulation $(n=20)$, CVIDio $=$ CVID with infections only $(\mathrm{n}=12), \mathrm{HC}=$ healthy controls $(n=12)$. Statistics: MannWhitney U-test. *p $<0.05$, $* * \mathrm{p}<0.01, * * * \mathrm{p}<0.001$. C $\%$ IFNg + CD $4+$ T-cells correlate with serum levels of CXCL9, CXCL10, and CXCL11. Statistics: Spearman correlation
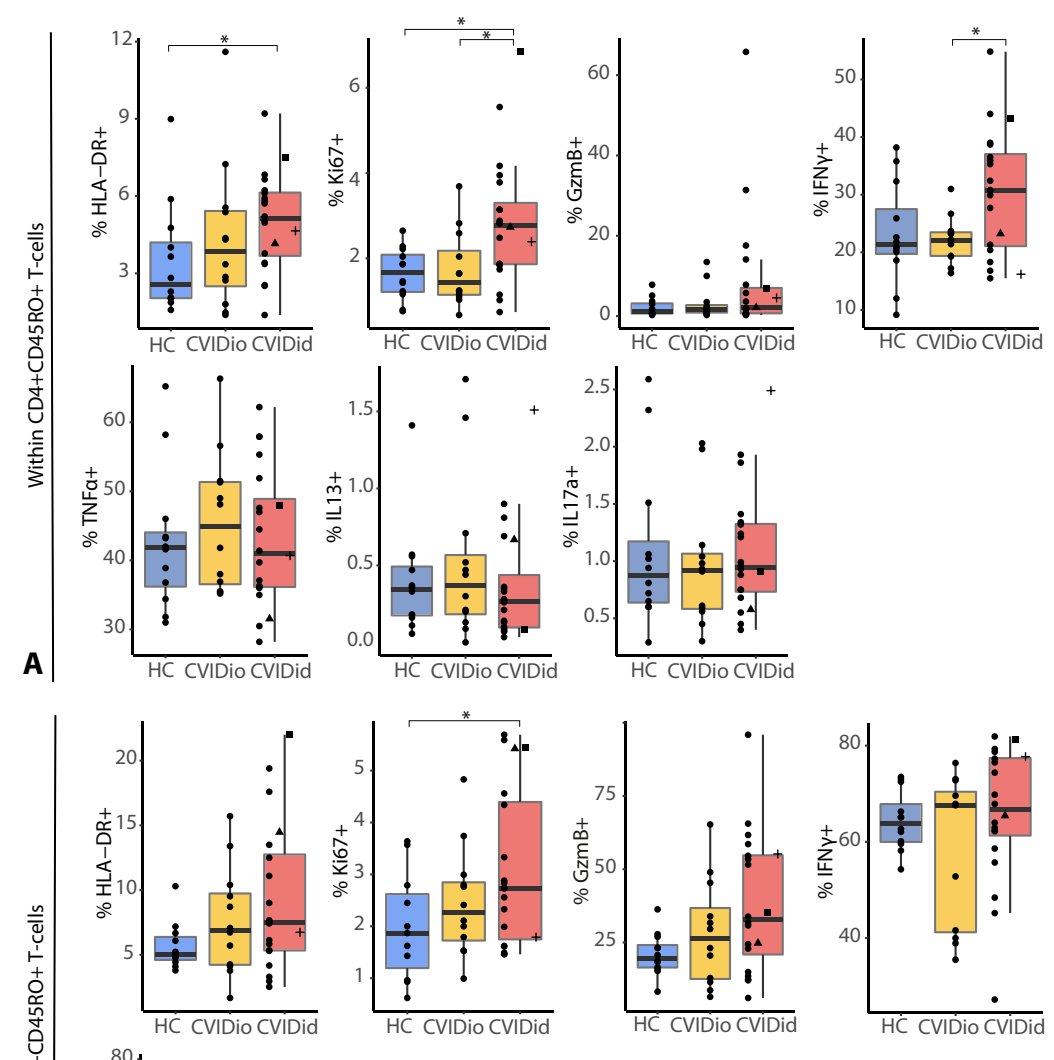

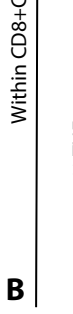

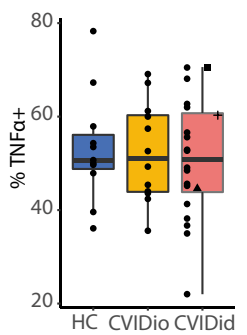

$$
\begin{array}{ll}
- & \text { HC } \\
\text { CVIDio } \\
\text { - CVIDid } \\
\square \text { STAT1 } \\
\Delta \text { CTLA4 } \\
+\quad \text { PIK3R1 }
\end{array}
$$

C
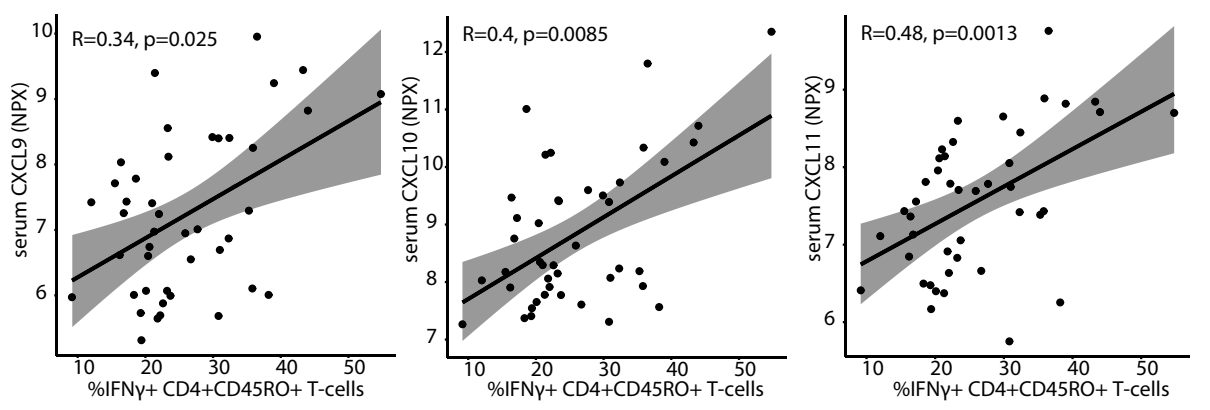

CD8 + T-cells, LAG3, CTLA4, ICOS, and TIGIT, but not PD1 and CD95, were similarly increased in CVIDid (Supplementary Fig. 8).

In order to assess whether the PD1 and LAG3 expressing cells were functionally exhausted, production of IFN $\gamma, \mathrm{GzmB}, \mathrm{Ki67}$, and CD95 was compared between the PD1/LAG3 + and PD1/LAG3 - populations (Fig. 3C and Supplementary Fig. 9). In CD4 + T-cells, the PD1 + and LAG3 + subpopulations showed equal or increased expression of IFN $\gamma$, Ki67, and/or GzmB as the
PD1 - and LAG3 - subpopulations, indicating retained functional capacity. Expression of PD1 correlated with serum levels of pro-inflammatory CXCL9, 10, and 11 as well as immune regulatory IL10 (Fig. 3D), further illustrating the association between PD1 and chronic inflammation. In CD $8+\mathrm{T}$-cells, IFN $\gamma$ and $\mathrm{GzmB}$ expression was also intact in PD1 + and LAG3 + cells, but Ki67 + cells were less frequent in the LAG3 + subpopulation, indicating reduced proliferative capacity (Supplementary Fig. 10). 

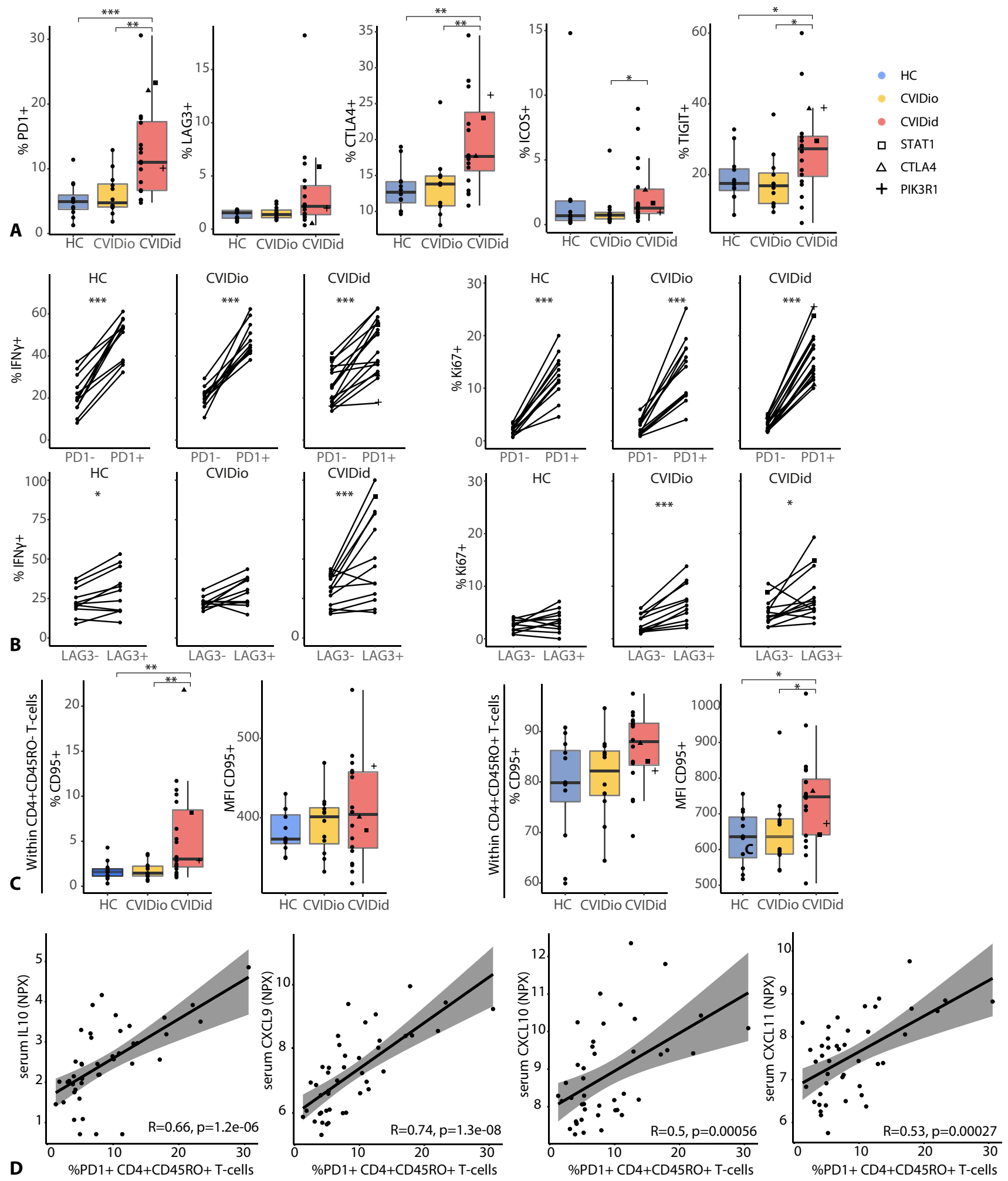

Fig. 3 Negative regulators of immune activation in CVID. A Proportions of PD1, LAG3, CTLA4, ICOS, and TIGIT in CD4 + CD45RO+T-cells. B Percentage and median fluorescence intensity of CD95 (FAS-L) in naïve $(\mathrm{CD} 45 \mathrm{RO}-)$ and effectormemory $(\mathrm{CD} 45 \mathrm{RO}+) \mathrm{CD} 4+\mathrm{T}$-cells. C Comparison of IFN $\gamma+$ and Ki67+cells in PD1- and LAG3-positive and negative populations. Only samples with $>50$ events in the PD1/LAG3-positive

and PD1/LAG3-negative populations were included. D Spearman correlation between PD1 and IL10, CXCL9, CXCL10, or CXCL11. CVIDid=CVID with immune dysregulation $(n=20)$, CVIDio $=$ CVID with infections only $(\mathrm{n}=12), \mathrm{HC}=$ healthy controls $(n=12)$. Statistics $(A \& C)$ : Mann-Whitney U-test. Statistics B: paired Wilcoxon-Rank test. $* \mathrm{p}<0.05, * * \mathrm{p}<0.01, * * * \mathrm{p}<0.001$ 


\section{CVIDid Regulatory T-cells Fail to Upregulate CTLA4}

In addition to negative regulators of immune activation, regulatory $\mathrm{T}$ (Treg) cells also contribute to limiting inflammation-related pathology. In contrast to previous studies [25], we did not observe a decreased proportion of CD25 + FOXP3 + Tregs in CVIDid, and a subgroup of CVIDid patients had even increased Treg frequencies (Fig. 4A). ICOS expression in CVIDid Tregs also indicated normal-to-increased activation [26] of Tregs (Fig. 4B), but CTLA4 expression was reduced in CVIDid Tregs, both in cell frequency as MFI in the CTLA4-positive population (Fig. 4B). The failure to upregulate CTLA4 in CVIDid Tregs was isolated to the activated and usually highly suppressive [27] CD27 + Treg population (Fig. 4C), and may result in reduced Treg function, as expression of CTLA4 is important for the suppressive function of Tregs [28]. In addition, a subpopulation of CVIDid patients showed low levels of TIGIT-expressing Tregs, which may impair TIGIT-driven selective suppression of Th1 and Th17 effector cells [29].

\section{Increased Migratory Capacity in CVIDid T-cells}

The immune dysregulation of most CVIDid patients occurs not only in the systemic immune system, but also locally in affected tissues, which are often mucosal sites (lung and/ or gut). Expression of the mucosal homing marker CCR9 was increased on naïve $\mathrm{CD} 4+$ and $\mathrm{CD} 8+$ populations of CVIDid T-cells (Fig. 5A), but not on non-naïve (CD45RA -) CD4+ T-cells (Fig. 5B), suggesting that this upregulation
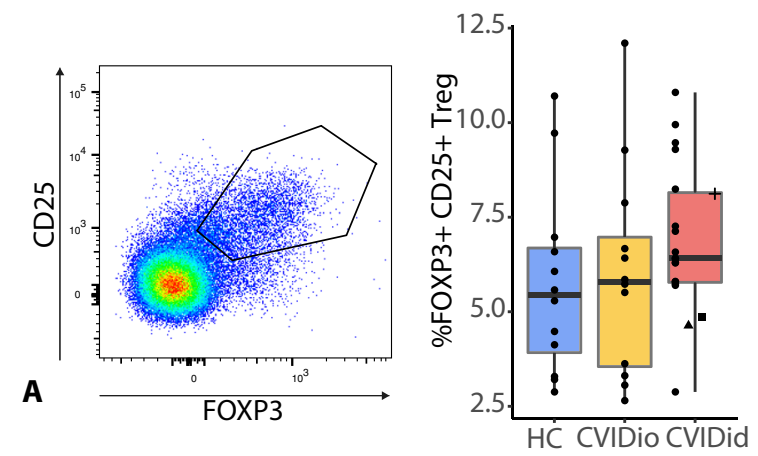

$$
\begin{array}{ll}
\text { - } & \text { HC } \\
& \text { CVIDio } \\
- & \text { CVIDid } \\
\square & \text { STAT1 } \\
\Delta & \text { CTLA4 } \\
+ & \text { PIK3R1 }
\end{array}
$$
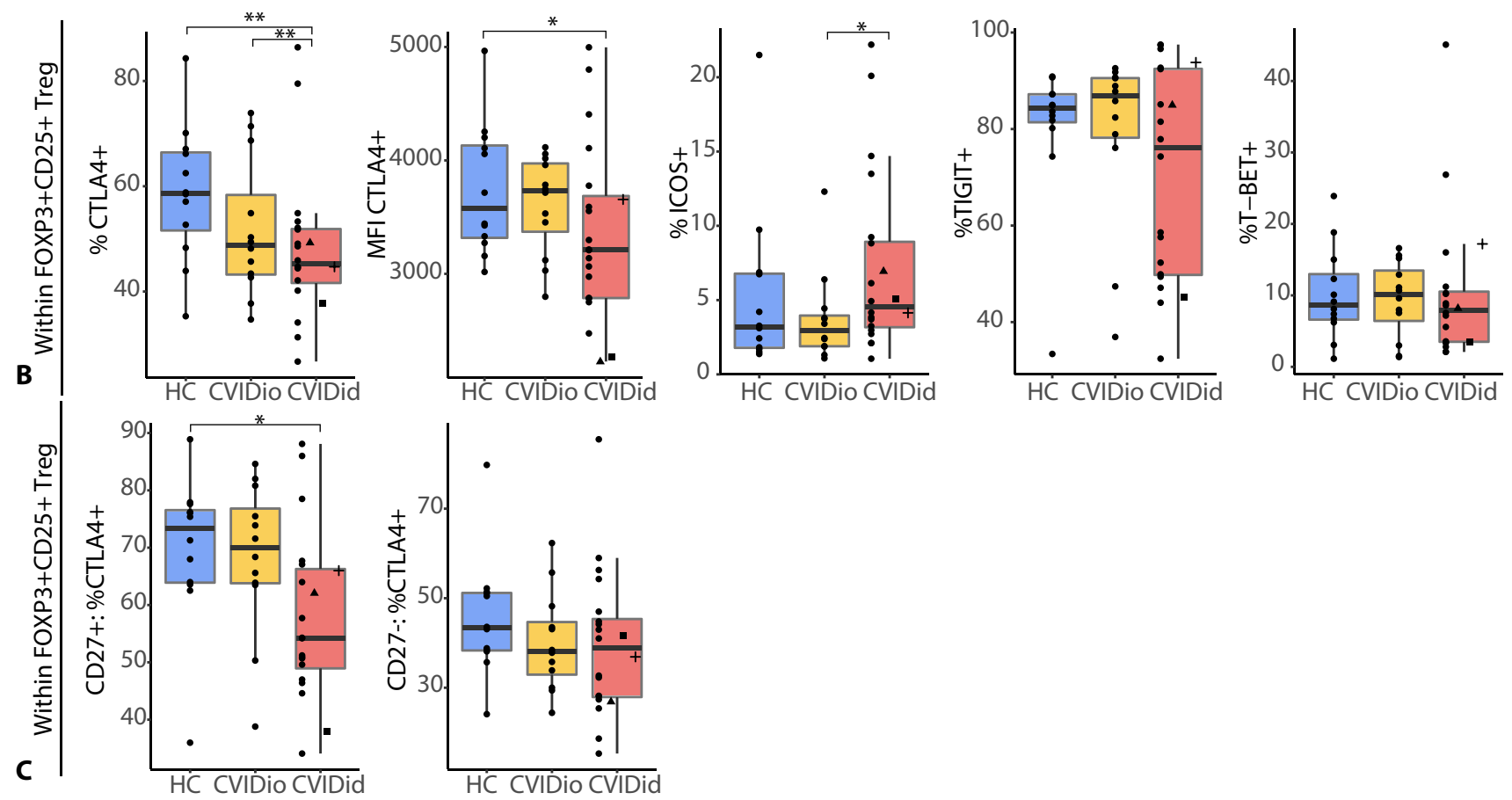

Fig. 4 Regulatory T-cells in CVID. A Gating strategy and proportion of $\mathrm{CD} 25+\mathrm{FOXP} 3+\mathrm{T}$-cells within $\mathrm{CD} 4+$ population. B Expression of CTLA4, ICOS, TIGIT, and T-BET in the Treg population. C Decreased fraction of CTLA4+ Tregs was confined to the
CD27 + population. CVIDid = CVID with immune dysregulation $(n=20)$, CVIDio $=$ CVID with infections only $(n=12), H C=$ healthy controls $(\mathrm{n}=12)$. Statistics: Mann-Whitney U-test. $* \mathrm{p}<0.05$, $* * \mathrm{p}<0.01, * * * \mathrm{p}<0.001$ 
was at least not entirely antigen-driven. CVIDid CD8 + nonnaïve (CD45RA -) T-cells (Fig. 5C) did also show increased expression of CCR9 as well as integrin $\alpha 4 \beta 1$, which mediates homing to inflamed tissues, including the lung. CVIDid FOXP3 + CD4 + T-cells (Fig. 5D) also showed increased migratory capacity, as they were enriched for cells expressing integrin $\alpha 4 \beta 1$ and gut-homing marker integrin $\alpha 4 \beta 7$.

\section{Patterns of T-cell Dysregulation in CVIDid Patients with GLILD}

Finally, in order to illustrate patterns of T-cell immune dysregulation in individual patients with varying severity of specific immune dysregulation phenotypes, we selected markers most strongly associated with (chronic) immune activation in CD4+ T-cells (HLA-DR, Ki67, IFN $\gamma$, and IFN $\gamma$ in PD1 + and $\mathrm{LAG} 3+, \mathrm{PD} 1+$, LAG3 +, TEMRA, and CD95) and immune regulation (Tregs, CTLA4 in Tregs, MFI of CTLA4 in Tregs, TIGIT in Tregs, ICOS in Tregs, and CTLA4 in CD4 + T-cells) in CVIDid. We investigated these in five patients with GLILD (Fig. 6), ranging from stably mild radiographic GLILD without lung function deterioration (patients 1 and 2), to clinical GLILD that required immunosuppressive treatment shortly after sampling (patients 4 and 5). In patients $1-4$, immune activation increased with severity of disease. In patient 3, who at the time of sampling had subclinical GLILD that exacerbated 3 years later, as well as patients 4 and 5, the median fluorescence intensity of CTLA4 on Tregs decreased, which was not observed for the two subclinical GLILD patients that remained stable ( 1 and 2). Patient 4 with STAT1 GoF, who required treatment for GLILD shortly after sampling, showed high levels of immune activation and PD1 expression, and low Treg functional markers. Finally, patient 5 with CTLA4 haploinsufficiency demonstrated low CTLA4 expression on Tregs as expected, but also low T-cell activation (HLA-DR, Ki67, and IFN $\gamma$ ) in peripheral blood, despite progressive GLILD and the need for treatment shortly after sampling.

\section{Discussion}

In this study, we showed that T-cells in patients with CVIDid were more often activated, proliferating, and Th1-skewed than those of patients with CVIDio. In addition, more CVIDid T-cells expressed immune co-inhibitory receptors PD1, LAG3, CTLA4, ICOS, and TIGIT, and these cells retained their inflammatory properties. Chronic activation was observed in both the CD4 + and CD8 + compartment. In the Treg compartment, we observed low CTLA4 expression in CVIDid, while ICOS expression remained intact. Finally, CVIDid T-cells showed increased migratory capacities to mucosal tissues.
High T-cell activation and Th-1 skewing in CVIDid were consistent with previous studies $[12,18]$. In addition, we did not observe increased frequencies of IL17-producing T-cells, despite our previous finding of increased IL$17 \mathrm{a}$ in serum of the same patients sampled at the same time. This supports the hypothesis for an alternative source of IL17a production in CVIDid, such as type-3 ILCs [30].

Previous studies have also reported increased PD1 expression in CVID, and interpreted this as a sign of functional exhaustion and impaired T-cell function [15, $18,31]$. However, we observed that the PD1- and LAG3expressing cells in CVIDid retained the capacity to produce pro-inflammatory cytokines and to proliferate, and thus were not functionally exhausted. Upregulation of negative regulators of co-stimulation has been suggested to be a mechanism to limit inflammation-related damage to tissues in settings of chronic inflammation, while maintaining the ability to respond to pathogens [32]. In CVIDid, however, it is possible that this compensatory response is insufficient in severe states of immune dysregulation and that these chronically activated cells still contribute to immune dysregulation-related pathology.

In addition to this chronically activated T-cell state, we observed a decreased ability of Tregs to upregulate CTLA4, while CTLA4 expression was increased in the whole CD $4+$ population. Expression of CTLA4 by Tregs is an important mechanism by which Tregs mediate their suppressive function [33]. Clinical CTLA4 haploinsufficiency often results in a CVIDid phenotype with hypogammaglobulinemia and autoimmune disease, and functional Treg dysfunction has been described [19]. In this study, the T-cell profile of the patient with CTLA4 haploinsufficiency was often not very different from the other non-genetic CVIDid patients. Therefore, the expression of CTLA4 in Tregs of non-genetic CVIDid patients may be relevant to the overall underlying pathophysiology of CVIDid and warrants further research. A recent study shows that abatacept, a CTLA4 fusion protein, was safe and effective in the treatment of CVIDid with interstitial lung disease [34]. As the population of CVIDid patients with low CTLA4 + CD27 + Tregs represented a mix of patients with pulmonary inflammation but also other organ-specific autoimmunity (data not shown), abatacept may be efficacious in other CVIDid patients as well. In addition, longitudinal monitoring of CTLA4 Treg expression in CVIDid may indicate whether it can be used as a biomarker for disease exacerbation and/or therapeutic response.

Despite these overall differences between CVIDid and CVIDio, the heterogeneity within the CVIDid group was substantial. Subgroup analyses of patients with organspecific autoimmunity did not yield insightful patterns, except that GLILD patients were often more extreme in 
Fig. 5 Migratory capacity in CVID: $\mathbf{A}$ in CCR7 + CD45RA + naïve CD4 and CD8 + T-cells, $\mathbf{B}$ in nonnaïve CD45RA - CD4 + T-cells, $\mathrm{C}$ in non-naïve CD45RA - CD8 + T-cells, D in FOXP3 + CD4 + T-cells. CVIDid $=$ CVID with immune dysregulation $(n=20)$, CVIDio $=$ CVID with infections only $(\mathrm{n}=12), \mathrm{HC}=$ healthy controls $(n=12)$. Statistics: MannWhitney U-test. ${ }^{*} \mathrm{p}<0.05$, $* * \mathrm{p}<0.01, * * * \mathrm{p}<0.001$
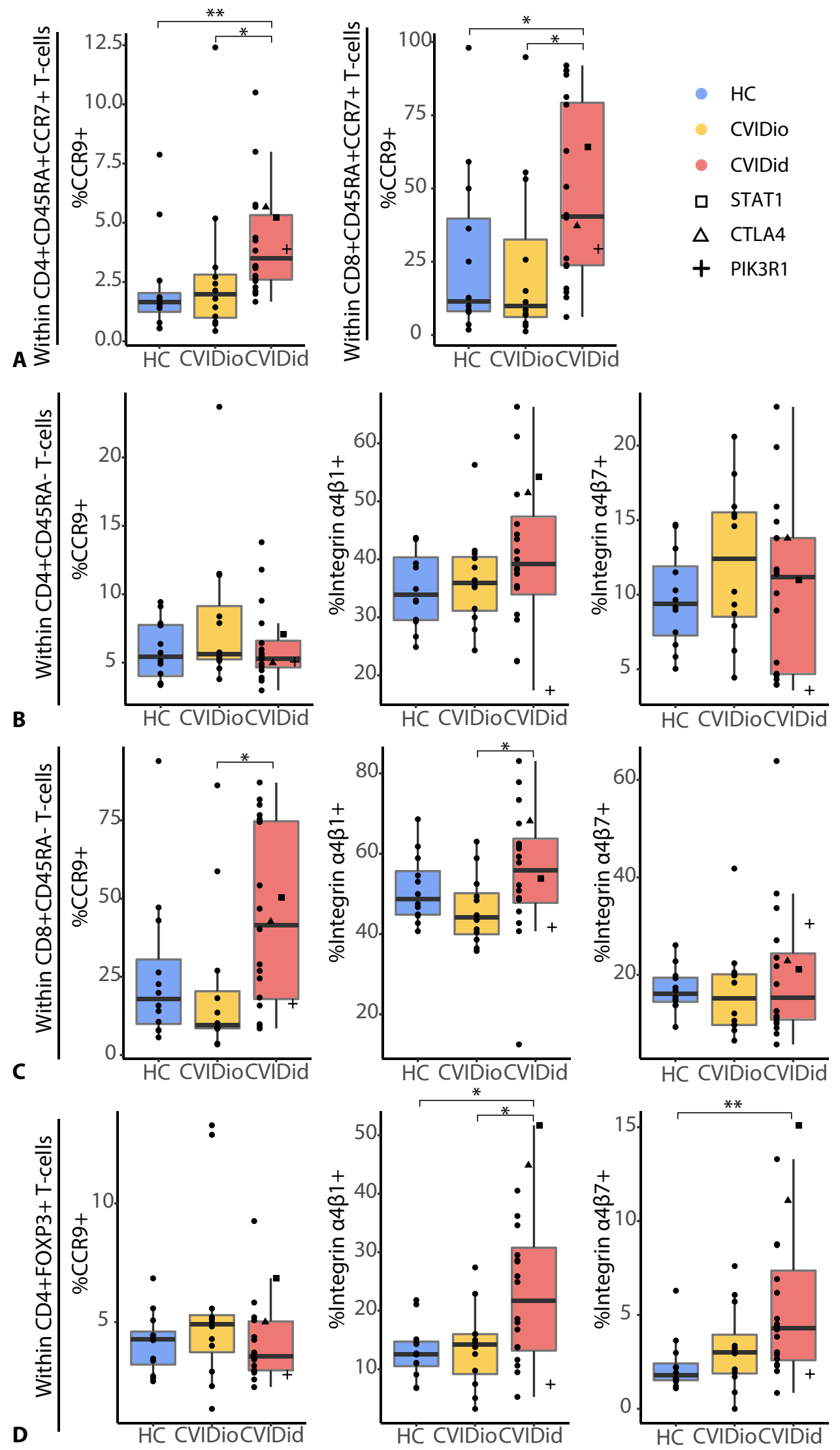


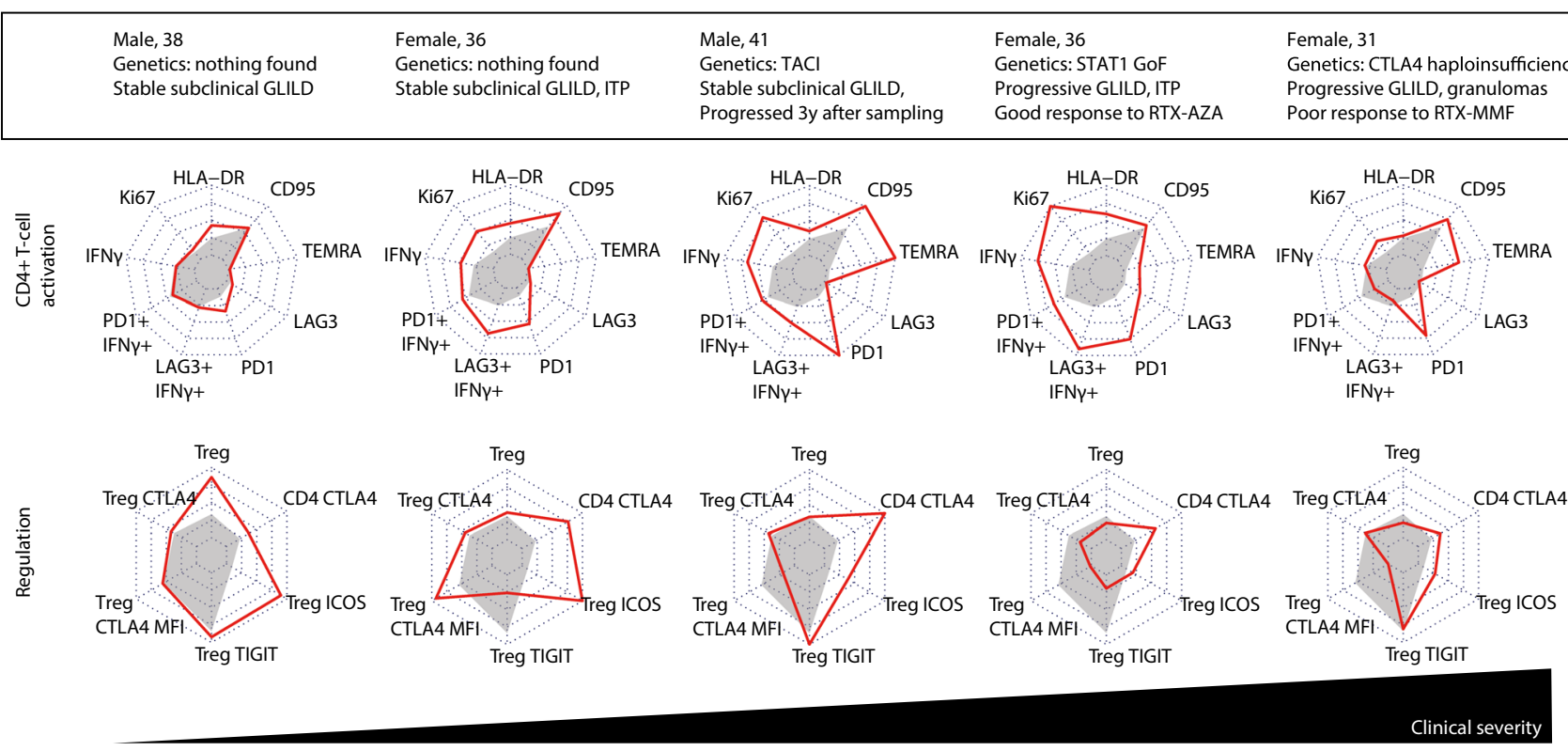

Fig. 6 Patterns of CD4+T-cell activation: \% of HLA-DR, Ki67, IFNg, IFNg in the PD1+population and the LAG3 + population, $\%$ PD1, \%LAG3，\%CD45RA + CCR7 - (TEMRA) cells, and \% of CD95. And patterns of T-cell regulation: \%CD25+FOXP3 + Treg, \%CTLA4 in Treg, MFI of CTLA4 in Treg, \% TIGIT in Treg, \%ICOS in Treg, \%CTLA4 in CD4+T-cells in five patients with GLILD. Red line indicates individual patient legends, gray-shaded area indicates

all observed differences (data not shown). In addition, disease severity did not always reflect immune activation. For example, the patient with CTLA4 haploinsufficiency did not show an inflammatory state in peripheral blood, while the patient that had the strongest IFN $\gamma$ signature combined with low immune regulation markers (Supplementary Fig. 11) was clinically stable and did not require immunosuppressive therapy. It is possible that the peripheral blood immune phenotype only gives limited information that is clinically relevant, as T-cells may have migrated to the inflamed tissues in the sickest patients. In addition, this study may be limited by sample size to detect differences between subgroups of CVIDid. One other aspect that this study does not address is differences in absolute T-cell numbers. A recent study showed that while absolute T-cells are often lower in CVID, they do not differ between CVIDio and CVIDid [13].

To conclude, these results indicate that CVIDid Th cells are highly activated, and that, unlike in classically exhausted cells such as originally described in chronic viral infection and cancer [32], PD1 and LAG3 expression in CVIDid CD4 + T-cells reflects chronic activation with preserved inflammatory potential rather than functional exhaustion, similar to previous findings in human auto-immune inflammation [24]. Combined studies of T-cell dysfunction and circulating inflammatory proteins the median for the CVIDio group. Axis ranges are the minimum and the maximum for that marker for the entire cohort. MFI, median fluorescence intensity; GLILD, granulomatous-lymphocytic interstitial lung disease; VUS, variant of unknown significance; ITP, idiopathic thrombocytopenia purpura; RTX, rituximab; AZA, azathioprine; MMF, mycophenolate mofetil; SCT, stem-cell transplantation; GOF, gain of function

in peripheral blood may help predict response to T-celltargeted therapies in individual CVIDid patients. Moreover, loss of CTLA4 upregulation in activated CVIDid Tregs may be mechanistically important in maintaining the inflammatory loop in CVIDid, and warrants further research.

Supplementary Information The online version contains supplementary material available at https://doi.org/10.1007/s10875-021-01084-6.

Author Contribution Study was conceived by HL, FvW, and RB. Sample collection was performed by RB, JM, PH, VD, and PE. Wet lab work was performed by RB and MvdW. Data analysis and interpretation: HL, FvW, and RB. All authors substantially contributed to the acquisition, analysis, or interpretation of the data, and all approved the final manuscript.

Funding This study was financially supported by the Wilhelmina Children's Hospital Fund (Utrecht, the Netherlands).

Data Availability Not applicable.

\section{Declarations}

Ethics Approval Ethical approval for this study for all participants was received from the Medical Ethical Committee of the Erasmus MC University Medical Center Rotterdam, the Netherlands (METC 2013-026). Written informed consent was obtained from all patients and controls according to the Declaration of Helsinki. 
Conflict of Interest PH reports research grants and personal fees from Shire/Takeda and CSL Behring. VD reports research grants and personal fees from Shire/Takeda, Griffols, Actelion, Novartis, Pharming, and CSL Behring. JM reports personal fees from Shire/Takeda. HL reports research grants from Shire/Takeda. All other authors declare no competing interests.

Open Access This article is licensed under a Creative Commons Attribution 4.0 International License, which permits use, sharing, adaptation, distribution and reproduction in any medium or format, as long as you give appropriate credit to the original author(s) and the source, provide a link to the Creative Commons licence, and indicate if changes were made. The images or other third party material in this article are included in the article's Creative Commons licence, unless indicated otherwise in a credit line to the material. If material is not included in the article's Creative Commons licence and your intended use is not permitted by statutory regulation or exceeds the permitted use, you will need to obtain permission directly from the copyright holder. To view a copy of this licence, visit http://creativecommons.org/licenses/by/4.0/.

\section{References}

1. Immunodeficiencies ES for Diagnostic Criteria PID. 2019. Available from: https://esid.org/Education/Diagnostic-Criteria-PID. Accessed 07-04-2021.

2. Cunningham-Rundles C. How I treat common variable immune deficiency. Blood. 2010;116:7-15.

3. Gathmann B, Mahlaoui N, Gérard L, Oksenhendler E, Warnatz $\mathrm{K}$, Schulze I, et al. Clinical picture and treatment of 2212 patients with common variable immunodeficiency. J Allergy Clin Immunol. 2014;134:116-26.

4. Chapel H, Lucas M, Lee M, Bjorkander J, Webster D, Grimbacher $\mathrm{B}$, et al. Common variable immunodeficiency disorders: division into distinct clinical phenotypes. Blood. 2008;112:277-87.

5. Resnick ES, Moshier EL, Godbold JH, Cunningham-Rundles C. Morbidity and mortality in common variable immune deficiency over 4 decades. Blood. 2012;119:1650-8.

6. Odnoletkova I, Kindle G, Quinti I, Grimbacher B, Knerr V. The burden of common variable immunodeficiency disorders: a retrospective analysis of the European Society for Immunodeficiency (ESID) registry data. Orphanet J Rare Dis. 2018;0:1-17.

7. Maarschalk-Ellerbroek LJ, Hoepelman AIM, Van Montfrans JM, Ellerbroek PM. The spectrum of disease manifestations in patients with common variable immunodeficiency disorders and partial antibody deficiency in a university hospital. J Clin Immunol. 2012;32:907-21.

8. Hurst JR, Verma N, Lowe D, Baxendale HE, Jolles S, Kelleher P, et al. British Lung Foundation/United Kingdom Primary Immunodeficiency Network consensus statement on the definition, diagnosis, and management of granulomatous-lymphocytic interstitial lung disease in common variable immunodeficiency disorders. J Allergy Clin Immunol Pract . Elsevier Inc; 2017;5:938-45. https:// doi.org/10.1016/j.jaip.2017.01.021

9. Gereige JD, Maglione PJ. Current understanding and recent developments in common variable immunodeficiency associated autoimmunity. Front Immunol. 2019;10:0-1.

10. Rao N, MacKinnon AC, Routes JM. Granulomatous and lymphocytic interstitial lung disease: a spectrum of pulmonary histopathologic lesions in common variable immunodeficiency-histologic and immunohistochemical analyses of 16 cases. Hum Pathol. Elsevier Inc.; 2015;46:1306-14. https://doi.org/10.1016/j.humpa th.2015.05.011
11. Chase NM, Verbsky JW, Hintermeyer MK, Waukau JK, TomitaMitchell A, Casper JT, et al. Use of combination chemotherapy for treatment of granulomatous and lymphocytic interstitial lung disease (GLILD) in patients with common variable immunodeficiency (CVID). J Clin Immunol. 2013;33:30-9.

12. Giovannetti A, Pierdominici M, Mazzetta F, Marziali M, Renzi C, Mileo AM, et al. Unravelling the complexity of T cell abnormalities in common variable immunodeficiency. J Immunol. 2007; 178:3932-43

13. Edwards ESJ, Bosco JJ, Aui PM, Stirling RG, Cameron PU, Chatelier J, et al. Predominantly antibody-deficient patients with non-infectious complications have reduced naive $\mathrm{B}$, Treg, Th17, and Tfh17 cells. Front Immunol. 2019;10:2593.

14. Unger S, Seidl M, van Schouwenburg P, Rakhmanov M, Bulashevska A, Frede N, et al. The TH1 phenotype of follicular helper T cells indicates an IFN- $\gamma$-associated immune dysregulation in patients with CD21low common variable immunodeficiency. J Allergy Clin Immunol . Elsevier Ltd; 2018;141:730-40. https://doi.org/10.1016/j.jaci.2017.04.041

15. Perreau M, Vigano S, Bellanger F, Pellaton C, Buss G, Comte $\mathrm{D}$, et al. Exhaustion of bacteria-specific CD4 T cells and microbial translocation in common variable immunodeficiency disorders. J Exp Med. 2014;211:2033-45. https://doi.org/10.1084/ jem.20140039

16. Le Coz C, Bengsch B, Khanna C, Trofa M, Ohtani T, Nolan BE, et al. Common variable immunodeficiency-associated endotoxemia promotes early commitment to the $\mathrm{T}$ follicular lineage. J Allergy Clin Immunol. Elsevier Inc.; 2019;144:1660-73. https://doi.org/10.1016/j.jaci.2019.08.007

17. Berbers RM, Drylewicz J, Ellerbroek PM, van Montfrans JM, Dalm VASH, van Hagen PM, et al. Targeted proteomics reveals inflammatory pathways that classify immune dysregulation in common variable immunodeficiency. J Clin Immunol. 2020;41:362-73.

18. Hultberg J, Ernerudh J, Larsson M, Nilsdotter-Augustinsson $\AA$, Nyström S. Plasma protein profiling reflects TH1-driven immune dysregulation in common variable immunodeficiency. J Allergy Clin Immunol. 2020;146(2):417-28.

19. Kuehn HS, Ouyang W, Lo B, Deenick EK, Niemela JE, Avery DT, et al. Immune dysregulation in human subjects with heterozygous germline mutations in CTLA4. Science (80). 2014;345:1623-7.

20. Grimbacher B, Hutloff A, Schlesier M, Glocker E, Warnatz K, Dräger R, et al. Homozygous loss of ICOS is associated with adult-onset common variable immunodeficiency. Nat Immunol. 2003;4:261-8.

21. Team RC. R: A language and environment for statistical computing. R Foundation for Statistical Computing, Vienna, Austria. 2019. p. URL https://www.R-project.org/.

22. Arumugakani G, Wood PMD, Carter CRD. Frequency of treg cells is reduced in CVID patients with autoimmunity and splenomegaly and is associated with expanded CD21lo B lymphocytes. J Clin Immunol. 2010;30:292-300.

23. Tian Y, Babor M, Lane J, Schulten V, Patil VS, Seumois G, et al. Unique phenotypes and clonal expansions of human CD4 effector memory $\mathrm{T}$ cells re-expressing CD45RA. Nat Commun. 2017;8:1473.

24. Petrelli A, Mijnheer G, Hoytema Van Konijnenburg DP, Van Der Wal MM, Giovannone B, Mocholi E, et al. PD-1+CD8+ T cells are clonally expanding effectors in human chronic inflammation. J Clin Invest. 2018;128:4669-81

25. Fevang B, Yndestad A, Sandberg WJ, Holm AM, Müller F, Aukrust $\mathrm{P}$, et al. Low numbers of regulatory T cells in common variable immunodeficiency: association with chronic inflammation in vivo. Clin Exp Immunol. 2007;147:521-5. 
26. Vocanson M, Rozieres A, Hennino A, Poyet G, Gaillard V, Renaudineau S, et al. Inducible costimulator (ICOS) is a marker for highly suppressive antigen-specific $\mathrm{T}$ cells sharing features of TH17/TH1 and regulatory T cells. J Allergy Clin Immunol. 2010;126(2):280-9.

27. Hornero RA, Georgiadis C, Hua P, Trzupek D, He L, Qasim W, et al. CD70 expression determines the therapeutic efficacy of expanded human regulatory T cells. Commun Biol. Springer US; https://doi.org/10.1038/s42003-020-1097-8

28. Wing K, Onishi Y, Prieto-Martin P, Yamaguchi T, Miyara M, Fehervari Z, et al. CTLA-4 control over Foxp3+ regulatory T cell function. Science (80). 2008;322:271-5.

29. Anderson AC, Joller N, Kuchroo VK. Lag-3, Tim-3, and TIGIT: Co-inhibitory receptors with specialized functions in immune regulation. Immunity. Elsevier Inc.; 2016;44:989-1004

30. Cols M, Rahman A, Maglione PJ, Garcia-Carmona Y, Simchoni $\mathrm{N}$, Ko HBM, et al. Expansion of inflammatory innate lymphoid cells in patients with common variable immune deficiency. J Allergy Clin Immunol. Elsevier Ltd; 2016;137:1206-1215.e6. https://doi.org/10.1016/j.jaci.2015.09.013
31. Stuchlý J, Kanderová V, Vlková M, Heřmanová I, Slámová L, Pelák O, et al. Common variable immunodeficiency patients with a phenotypic profile of immunosenescence present with thrombocytopenia. Sci Rep. 2017;7:1-13.

32. Speiser DE, Utzschneider DT, Oberle SG, Münz C, Romero P, Zehn D. T cell differentiation in chronic infection and cancer: functional adaptation or exhaustion? Nat Rev Immunol. 2014;14:768-74.

33. Walker LSK. Treg and CTLA-4: two intertwining pathways to immune tolerance. J Autoimmun. Elsevier Ltd; 2013;45:49-57. https://doi.org/10.1016/j.jaut.2013.06.006

34. Spee-mayer C Von, Echternach C, Agarwal P, Gutenberger S. Abatacept use is associated with steroid dose reduction and improvement in fatigue and CD4-dysregulation in CVID patients with interstitial lung disease. J Allergy Clin Immunol Pract. Elsevier Inc; 2020;9:760-770.e10. https://doi.org/10.1016/j.jaip.2020. 10.028

Publisher's Note Springer Nature remains neutral with regard to jurisdictional claims in published maps and institutional affiliations. 